\title{
Pneumococci causing invasive disease in children prior to the introduction of pneumococcal conjugate vaccine in Scotland
}

Correspondence
Stuart C. Clarke
stuartcclarke@hotmail.com

Received 2 February 2006 Accepted 28 April 2006

\author{
Stuart C. Clarke, ${ }^{1,2,3} \dagger$ Johanna M. C. Jefferies, ${ }^{1}$ Andrew J. Smith, ${ }^{4}$ \\ Jim McMenamin, ${ }^{5}$ Timothy J. Mitchell ${ }^{2}$ and Giles F. S. Edwards ${ }^{1}$ \\ ${ }^{1}$ Scottish Meningococcus and Pneumococcus Reference Laboratory, Stobhill Hospital, \\ Balornock Road, Glasgow G21 3UW, UK \\ ${ }^{2}$ Division of Infection and Immunity, Institute of Biomedical and Life Sciences, University of \\ Glasgow, Glasgow G12 800, UK \\ ${ }^{3}$ Portsmouth City Teaching PCT, Milton Road, Portsmouth PO3 6DP, UK \\ ${ }^{4}$ Glasgow Dental School, 378 Sauchiehall Street, Glasgow G2 3JZ, UK \\ ${ }^{5}$ Health Protection Scotland, Clifton Place, Glasgow G3 7LN, UK
}

This study aimed to determine the serotypes and sequence types (STs) of pneumococci causing paediatric invasive disease in Scotland prior to the introduction of pneumococcal conjugate vaccines (PCVs). All invasive pneumococci isolated between 2000 and 2004 from children aged less than 5 years in Scotland were used. The isolates were characterized by serotyping and multi-locus sequence typing. Two hundred and seventeen pneumococci were characterized into 22 different serogroups/types, the most common, in rank order, being 14, 19F, 6B, 18C, $23 \mathrm{~F}, 9 \mathrm{~V}, 4,1,19 \mathrm{~A}$ and $6 \mathrm{~A}$. They were further genotyped into 77 different STs, the three most common being 9,162 and 176. Common serotypes possessed multiple STs, but pneumococci of a particular clone were mostly associated with a particular serotype. The seven most common serotypes are included in the 7-valent polysaccharide conjugate vaccine (PCV7). Serotype coverage for PCV7 was $76.5 \%$ in those aged less than 5 years but increased to $88.9 \%$ for those aged 1 year. The introduction of PCV7 into the childhood immunization schedule would reduce the burden of pneumococcal disease in children, although continued surveillance of invasive pneumococcal disease will be required before, during and after the introduction of PCVs.

\section{INTRODUCTION}

Pneumococcal infection causes substantial morbidity and mortality, especially in the young and old. Streptococcus pneumoniae (the pneumococcus) is a Gram-positive bacterium and is classified into more than 90 pneumococcal serotypes in 46 serogroups (Henrichsen, 1995). However, the majority of invasive and non-invasive disease is associated with a much smaller number of serotypes. The pneumococcus is one of a number of bacterial pathogens that are highly promiscuous; it is able to exchange DNA with members of its own and other related species by transformation. This means that genes encoding virulence factors, including the polysaccharide capsule, can be

†Present address: Hampshire and Isle of Wight Health Protection Unit, Oakley Road, Southampton SO16 4GX, UK.

Abbreviations: IPD, invasive pneumococcal disease; MLST, multi-locus sequence typing; PCV, pneumococcal conjugate vaccine; ST, sequence type. exchanged, leading to the existence of capsule switch (Coffey et al., 1991). This is important as, at present, available pneumococcal vaccines are based on pneumococcal capsular polysaccharide.

The prevention of invasive pneumococcal disease (IPD) by immunization is an attractive proposition and new pneumococcal vaccines have the potential to not only prevent a proportion of IPD, but also reduce the carriage of vaccine serotypes within which antibiotic resistance is most prevalent. The 7-valent pneumococcal conjugate vaccine (PCV7) was licensed in the UK in 2001 for use in children under 5 years of age within certain at-risk groups (Department of Health, 2002a, 2002b). PCV7 contains the polysaccharides of serotypes $4,6 \mathrm{~B}, 9 \mathrm{~V}, 14,18 \mathrm{C}, 19 \mathrm{~F}$ and $23 \mathrm{~F}$ conjugated to a non-toxic diphtheria variant carrier protein $\left(\mathrm{CRM}_{197}\right)$. Initial studies performed in the USA to assess the molecular epidemiology of the pneumococcus prior to and immediately following vaccine administration show that the use of PCV7 has significantly reduced the burden of 
pneumococcal disease in young children (Gertz et al., 2003; Whitney et al., 2003). It has also prevented a substantial proportion of carriage disease, thereby reducing the incidence of antibiotic-resistant pneumococci (Byington et al., 2002; McEllistrem et al., 2005; Stephens et al., 2005; Whitney et al., 2003).

A knowledge of pneumococcal disease epidemiology can provide important strategic data for deciding appropriate vaccine policy and vaccine formulation. In addition, in order to fully evaluate the impact of PCV7, there must be data available on the molecular epidemiology of the pneumococcus prior to the introduction of the vaccine. The aim of this study was therefore to establish the incidence of invasive pneumococcal disease in children less than 5 years old in Scotland prior to the introduction of PCV7. The serotype incidence and clonal distribution of these pneumococci was determined.

\section{METHODS}

Incidence of pneumococcal disease. The incidence of IPD in Scotland was determined using data from the Scottish enhanced pneumococcal surveillance programme (Kyaw et al., 2003). All cases of IPD from all National Health Service Board areas of Scotland between 2000 and 2004 were included in the study. The incidence of IPD in children less than 5 years of age was determined from this dataset. Age breakdowns of $<2$ months, $2-5$ months, 6-11 months, 1 year and 2-4 years were used and the incidence of IPD in each was calculated.

Pneumococcal isolates. All pneumococci isolated from those children less than 5 years of age with IPD identified above were used. Pneumococci were isolated and characterized in Scottish diagnostic microbiology laboratories and sent to the Scottish Meningococcus and Pneumococcus Reference Laboratory (SMPRL) as part of the enhanced pneumococcal surveillance programme in Scotland (Kyaw et al., 2003). All pneumococci were characterized at the SMPRL by serotyping and multi-locus sequence typing (MLST). Serotyping was performed by co-agglutination using reagents from the Statens Serum Institut, Denmark, as described by Smart (1986). For MLST, chromosomal DNA was prepared as described previously (Jefferies et al., 2003) and MLST was performed using the method of Enright \& Spratt (1998) but with an automated protocol (Jefferies et al., 2003). Briefly, internal fragments of seven housekeeping genes, aroE, $g d h, g k i, r e c P, s p i, x p t$ and $d d l$, were amplified using PCR and the nucleotide sequences determined on both strands using a 96-well format liquid-handling robot and an automated DNA sequencing system (Clarke et al., 2001; Jefferies et al., 2003). Alleles and sequence types (STs) for MLST were assigned with reference to the S. pneumoniae MLST database (http://spneumoniae.mlst.net) (Diggle \& Clarke, 2002). Novel profiles arising from the description of a new allele or new combination of alleles were submitted to the curator of the MLST database and assigned new STs. If there were any differences amongst serotypes of the same ST, the serotypes were rechecked.

Analysis of pneumococcal serotype and MLST data. Serotype and MLST data for all pneumococci isolated from children less than 5 years of age were analysed. The potential for serotype replacement or capsule switch was determined. The relationship between pneumococci of different STs was established using Based Upon Related Sequence Types (BURST) program analysis (Feil et al., 2004).

\section{RESULTS}

\section{Paediatric invasive pneumococcal disease}

A total of 238 pneumococci were isolated from cases of IPD in children less than 5 years of age in Scotland between January 2000 and August 2004. Serotyping of these pneumococci indicated that 21 were not serotypable and, for the purposes of this study, these were excluded from further analysis. A total of 217 pneumococci were therefore available for the study. One hundred and twenty-two pneumococci were from males, 89 from females and for 6 the sex of the patient was not provided. Fourteen cases occurred in patients aged less than 2 months of age, 22 in those aged between 2 and 5 months, 44 in those aged between 6 and 11 months, 99 in those aged 1 year and 38 in those aged between 2 and 4 years of age. Eleven cases occurred in patients less than 1 month of age.

\section{Pneumococcal serotypes}

Twenty-two different serogroups/types were found amongst the 217 isolates serotyped (Fig. 1). The ten most common serotypes accounted for $189(87 \cdot 1 \%)$ of all isolates in this study and, in rank order, were $14(36 \cdot 9 \%), 19 \mathrm{~F}(10 \cdot 1 \%), 6 \mathrm{~B}$ $(10 \cdot 1 \%), 18 \mathrm{C}(6 \cdot 0 \%), 23 \mathrm{~F}(5 \cdot 1 \%), 9 \mathrm{~V}(4 \cdot 6 \%), 4(3 \cdot 7 \%), 1$ $(3 \cdot 7 \%), 19 \mathrm{~A}(3 \cdot 7 \%)$ and $6 \mathrm{~A}(3 \cdot 2 \%)$. Those serotypes represented in PCV7 therefore accounted for $76.5 \%$ of all isolates $(n=166)$, which in numerical order are serotype 4 (3.7\%), 6B (10.1\%), 9V (4.6\%), $14(36 \cdot 9 \%), 18 \mathrm{C}(6 \cdot 0 \%)$, $19 \mathrm{~F}(10 \cdot 1 \%)$ and $23 \mathrm{~F}(5 \cdot 1 \%)$. The greatest number of different serotypes $(n=16)$ was seen in those aged $6-$ 11 months, indicating greater heterogeneity of pneumococci in this age group. The most common serotype was 14, accounting for $11(28.9 \%)$ isolates in those aged between 2 and 4 years, $44(44 \cdot 4 \%)$ in those aged 1 year, $15(34 \%)$ in those aged 6-11 months and $10(45 \cdot 5 \%)$ in those aged 2-5 months. There were no serotype 14 pneumococci in those aged less than 2 months, although the total number of pneumococci in this age group was only 15 . Serotypes $6 \mathrm{~B}$, $14,18 \mathrm{C}, 19 \mathrm{~F}$ and $23 \mathrm{~F}$ were common in those aged $1-2$ years, accounting for $83(84 \%)$ of all isolates in this age group.

\section{Sequence types}

Using MLST, all isolates were characterized into 77 different STs (Table 1). There were 16 new STs, namely 1034, 1197, $1213,1214,1216,1238,1239,1240,1241,1246,1253,1254$, $1256,1303,1359$ and 1384. In rank order, the three most common STs were $9(n=55), 162(n=18)$ and $176(n=15)$. These accounted for $40.5 \%$ of all isolates in this study. The pneumococci in this study were therefore highly diverse. BURST analysis indicated 16 related groups and a total of 24 singleton STs. The three most common STs did not cluster together and were therefore not closely related.

\section{Relationship between serotype and ST}

Common serotypes possessed multiple STs and those associated with the serotypes present in PCV7 are shown 


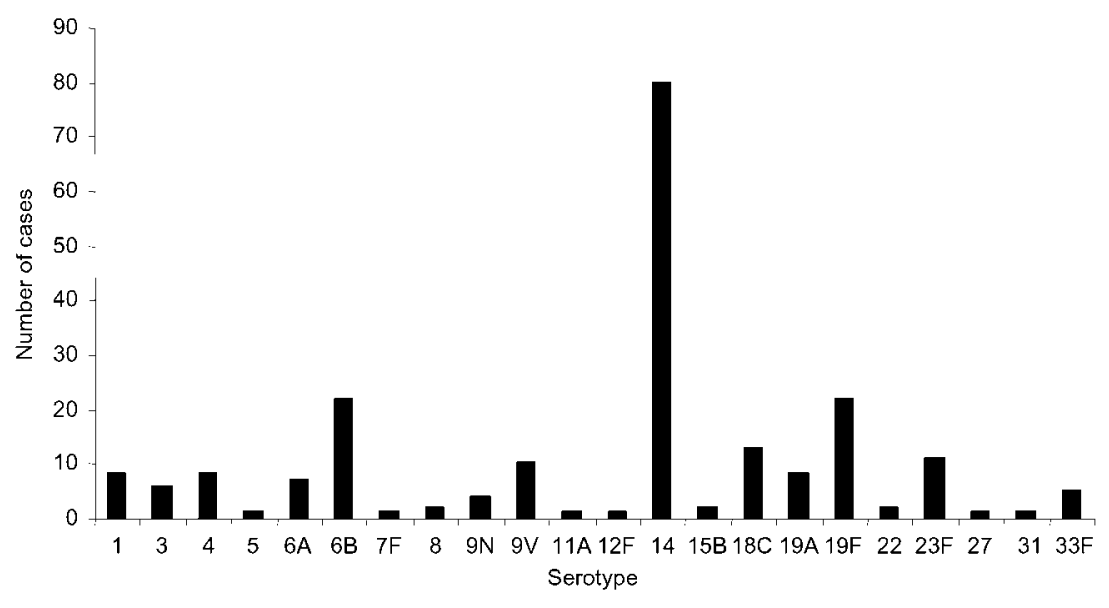

Fig. 1. Pneumococcal serotypes amongst those aged less than 5 years in Scotland, 2000-2004. in Table 2. Twelve STs were associated with the most common serotype in the study, serotype 14, although the most common STs for this serotype were 9 and 124. For serotype 19F, ST162 was the most common; for serotype 18C, ST113 was the most common; for serotype 6B, ST176 was the most common; for serotype 23F, ST311 was the most common; for serotype 9V, ST162 was the most common; and for serotype 4, ST206 was the most common. There was no indication of the same ST being present in serotypes within the same serogroup (i.e. serotype $9 \mathrm{~V}$ within serogroup 9).

Table 1. Pneumococcal STs found in this study

\begin{tabular}{|lrrrrrrr|}
\hline ST & $\boldsymbol{n}$ & ST & $\boldsymbol{n}$ & ST & $\boldsymbol{n}$ & ST & $\boldsymbol{n}$ \\
\hline 9 & 55 & 3 & 1 & 402 & 1 & 1034 & 1 \\
162 & 18 & 15 & 1 & 419 & 1 & 1197 & 1 \\
176 & 15 & 36 & 1 & 444 & 1 & 1213 & 1 \\
124 & 14 & 40 & 1 & 458 & 1 & 1214 & 1 \\
113 & 8 & 53 & 1 & 469 & 1 & 1216 & 1 \\
311 & 8 & 62 & 1 & 571 & 1 & 1233 & 1 \\
199 & 7 & 63 & 1 & 629 & 1 & 1238 & 1 \\
180 & 4 & 81 & 1 & 638 & 1 & 1239 & 1 \\
206 & 4 & 96 & 1 & 673 & 1 & 1240 & 1 \\
65 & 3 & 110 & 1 & 681 & 1 & 1241 & 1 \\
100 & 3 & 139 & 1 & 688 & 1 & 1246 & 1 \\
306 & 3 & 156 & 1 & 717 & 1 & 1253 & 1 \\
405 & 3 & 165 & 1 & 813 & 1 & 1254 & 1 \\
426 & 3 & 177 & 1 & 815 & 1 & 1256 & 1 \\
138 & 2 & 191 & 1 & 825 & 1 & 1303 & 1 \\
205 & 2 & 218 & 1 & 834 & 1 & 1384 & 1 \\
227 & 2 & 236 & 1 & 839 & 1 & 1359 & 1 \\
246 & 2 & 271 & 1 & 864 & 1 & & \\
409 & 2 & 304 & 1 & 944 & 1 & & \\
433 & 2 & 315 & 1 & 999 & 1 & & \\
& & & & & & & \\
\hline
\end{tabular}

\section{Relationship between ST and serotype}

Isolates of a particular clone were, more often than not, associated with a particular serotype (Table 3). Of the 20 different STs with two or more isolates, 16 were of single serotypes. These included the common ST9 and ST124 clones. However, four STs were associated with more than one serotype (Table 4). These were STs 138, 162, 176 and

Table 2. STs represented in serotypes present in the PCV7 vaccine

\begin{tabular}{|ll|}
\hline Serotype & \multicolumn{1}{c|}{ ST } \\
\hline 4 & $205,206,246$ \\
$6 \mathrm{~B}$ & $176,1034,1240,1256$ \\
$9 \mathrm{~V}$ & $156,162,834,999$ \\
14 & $3,9,15,124,138,409,629,1213,1214,1241$, \\
& 1246,1254 \\
$18 \mathrm{C}$ & $63,110,113,638,1238,1303$ \\
$19 \mathrm{~F}$ & $162,165,177,236,271,426,688,839,1233,1359$ \\
$23 \mathrm{~F}$ & $36,40,311,825$ \\
& \\
\hline
\end{tabular}

Table 3. STs associated with more than one isolate but with single serotypes

\begin{tabular}{|lccc|}
\hline ST & Serotype $(\boldsymbol{n})$ & ST & Serotype $(\boldsymbol{n})$ \\
\hline 9 & $14(55)$ & 227 & $1(2)$ \\
65 & $6 \mathrm{~A} \mathrm{(3)}$ & 246 & $4(2)$ \\
100 & $33 \mathrm{~F}(3)$ & 306 & $1(3)$ \\
113 & $18 \mathrm{C}(8)$ & 311 & $23 \mathrm{~F}(8)$ \\
124 & $14(14)$ & 405 & $9 \mathrm{~N}(3)$ \\
180 & $3(4)$ & 409 & $14(2)$ \\
205 & $4(2)$ & 426 & $19 \mathrm{~F}(3)$ \\
206 & $4(4)$ & 433 & $22 \mathrm{~F}(2)$ \\
\hline
\end{tabular}


Table 4. STs associated with more than one isolate and with multiple serotypes

\begin{tabular}{|ll|}
\hline ST & \multicolumn{1}{c|}{ Serotype $(\boldsymbol{n})$} \\
\hline 138 & $6 \mathrm{~B}(1), 14(1)$ \\
162 & $1(1), 9 \mathrm{~V}(6), 19 \mathrm{~F}(11)$ \\
176 & $6 \mathrm{~B}(14), 19 \mathrm{~A}(1)$ \\
199 & $15 \mathrm{~B}(1), 19 \mathrm{~A}(6)$ \\
\hline
\end{tabular}

199. New STs were associated with serotypes $6 \mathrm{~B}, 14,18 \mathrm{C}$ and $19 \mathrm{~F}$. For serotype $6 \mathrm{~B}$, there were three new STs, namely $1034,1240,1256$. For serotype 14, there were five new STs, $1213,1214,1241,1246$ and 1254; for serotype 18C, there were two new STs, 1238 and 1303; and for serotype 19F, there were two new STs, 1233 and 1359.

\section{DISCUSSION}

Recent studies in the UK have characterized collections of pneumococci in an attempt to understand better their clonal distribution, population biology and invasive disease potential. However, few data are available in the UK on the clonal distribution of a large set of pneumococci in children less than 5 years of age. It is important to understand the extent of the effect the new PCVs will have on IPD in the UK and, by characterizing a large collection of pneumococci causing disease in the young, an insight can be gained into the clonal distribution of IPD prior to the introduction of PCV7.

In this study, serotyping and MLST were used to characterize pneumococci collected from children of less than 5 years of age over a 5 year period. Serotyping provides an understanding of circulating capsule types but limited discrimination between individual clones, as a single serotype typically includes a number of genetically divergent clones (Brueggemann et al., 2003). Therefore, MLST was also performed, as this can be used to provide additional discrimination and information can be easily shared via the Internet. Using these two methods, the genetic relationship between particular clones within a serotype could be observed. This is important, as there is ample evidence that pneumococci can change serotype (Coffey et al., 1998, 1999; Meats et al., 2003; Nesin et al., 1998), as has also been observed in the meningococcus (Stefanelli et al., 2003; Swartley et al., 1997). The introduction of vaccines that only protect against selected serotypes, as is the case with PCV7, may result in serotype replacement or select for strains that have undergone capsule switch (Jefferies et al., 2004; Spratt \& Greenwood, 2000). In the former, clones of a particular ST may have the capacity to express different serotypes via the transformation (horizontal DNA exchange) of capsular genes. For example, in the USA, two recent reports describe the presence of related or identical clones with differing serotypes, namely $19 \mathrm{~A} / 19 \mathrm{~F}$ and $23 \mathrm{~A} / 23 \mathrm{~F}$ (Cordeiro et al., 2005; Pai et al., 2005). However, it may be that PCV7 provides vaccine-related serotype coverage for these clones, although further data are required before satisfactory conclusions can be made. In serotype replacement, serotypes not included in the PCV would replace the niche left by those covered by the PCV. The latter may be more important and there is a fear that rates of disease caused by nonvaccine serotypes will increase. Nasopharyngeal carriage rates of non-PCV pneumococci may also increase among vaccinated children. Although initial studies evaluating the introduction of PCVs only observed an increase in non-PCV serotype in otitis media (Eskola et al., 2001), a more recent study has shown evidence of serotype replacement, although there was little clear evidence amongst disease isolates for serotype switching events driven solely by the selective pressure of PCV7 (Beall et al., 2006).

The incidence rate of IPD amongst children less than 5 years of age was in agreement with that reported for England and Wales between 1996 and 1998 (Miller et al., 2000) and the serotypes commonly associated with the pneumococci causing IPD were also similar to those seen in a previous study (McChlery et al., 2005). The results of this study should therefore be able to be generalized to the whole of the UK although, in the present study, the rank order of the serotypes was different, possibly due to the larger dataset used. Serotype coverage of PCV7 for those aged under 5 years was found to be higher than that previously reported in the UK (Clarke et al., 2004b; McChlery et al., 2005; Miller et al., 2000). The overall coverage was found to be $76.5 \%$ for children aged less than 5 years. Not surprisingly, serotype 14 was the most common serotype in this study, accounting for more than one-third of all pneumococci and being the most common serotype in all age groups. Serotype 14 is common in the UK and is associated with the presence of two major circulating clones, ST9 and ST124. Importantly, the seven most common serotypes found in this study are included in PCV7 although the next three most common serotypes are not. These latter serotypes, 1, 19A and 6A, together accounted for more than $10 \%$ of all cases of IPD in this study. This is an important observation, as serotype 1 is associated with a high attack rate and is one of the few serotypes associated with disease outbreaks (Brueggemann \& Spratt, 2003; Brueggemann et al., 2003). Serotypes 19A and 6A are as common as serotype 1 but have lower attack rates (Brueggemann et al., 2003).

It is not surprising that new STs were found in this study as relatively few large studies of collections of pneumococci have been performed, particularly in geographically distinct regions (Brueggemann et al., 2003; Enright \& Spratt, 1998; Hanage et al., 2005; Jefferies et al., 2004; McChlery et al., 2005; McGee et al., 2001). The isolates characterized to date clearly do not represent all clones currently circulating worldwide. The common serotypes were associated with multiple STs, as described in previous studies (Brueggemann et al., 2003; Clarke et al., 2004a; Jefferies et al., 2004; McChlery et al., 2005). In a previous study, it was found that serotype 14 pneumococci were genetically 
diverse, with as many as ten different STs causing invasive disease in Scotland (Clarke et al., 2004a). In this study, serotype 14 was associated with 12 STs and five of them were newly described. In contrast, clones were mostly associated with a particular serotype.

Although the extent of simultaneous carriage of different serotypes is not known, a limitation of this study, as well as most others reporting pneumococcal incidence data, is the possibility of simultaneous carriage of more than one pneumococcal serotype (Austrian, 1981, 1986; Chaves et al., 2003). However, the methods used in this study and the data resulting from it compare well with others. Moreover, the completeness of reporting of IPD in Scotland and the availability of pneumococci from such disease means that the data presented here is likely to be a good representation of the actual disease and serotype incidence of IPD in those less than 5 years of age in Scotland. Hence, the genetic relationship between different pneumococci described in this study is likely to be a good reflection of the current situation. Since those PCVs undergoing development may provide additional protection in adults, further studies will be required to establish the burden of disease in adults, as well as children. Nevertheless, this study provides a detailed insight into the serotypes and STs of pneumococci causing IPD in children under 5 years of age in Scotland and, as such, provides the baseline for continued surveillance after the introduction of PCV7.

\section{ACKNOWLEDGEMENTS}

The authors are grateful to all staff of the SMPRL for performing serotyping and MLST. This project made use of the Multi Locus Sequence Typing website (http://www.mlst.net) developed by ManSuen Chan and David Aanensen and funded by the Wellcome Trust.

\section{REFERENCES}

Austrian, R. (1981). Pneumococcus: the first one hundred years. Rev Infect Dis 3, 183-189.

Austrian, R. (1986). Some aspects of the pneumococcal carrier state. J Antimicrob Chemother 18 (Suppl. A), 35-45.

Beall, B., McEllistrem, M. C., Gertz, R. E., Jr, \& 9 other authors (2006). Pre- and postvaccination clonal compositions of invasive pneumococcal serotypes for isolates collected in the United States in 1999, 2001, and 2002. J Clin Microbiol 44, 999-1017.

Brueggemann, A. B. \& Spratt, B. G. (2003). Geographic distribution and clonal diversity of Streptococcus pneumoniae serotype 1 isolates. J Clin Microbiol 41, 4966-4970.

Brueggemann, A. B., Griffiths, D. T., Meats, E., Peto, T., Crook, D. W. \& Spratt, B. G. (2003). Clonal relationships between invasive and carriage Streptococcus pneumoniae and serotype- and clonespecific differences in invasive disease potential. J Infect Dis 187, 1424-1432.

Byington, C. L., Spencer, L. Y., Johnson, T. A. \& 8 other authors (2002). An epidemiological investigation of a sustained high rate of pediatric parapneumonic empyema: risk factors and microbiological associations. Clin Infect Dis 34, 434-440.
Chaves, F., Campelo, C., Sanz, F. \& Otero, J. R. (2003). Meningitis due to mixed infection with penicillin-resistant and penicillinsusceptible strains of Streptococcus pneumoniae. J Clin Microbiol 41, 512-513.

Clarke, S. C., Diggle, M. A. \& Edwards, G. F. S. (2001). Semiautomation of multilocus sequence typing for the characterization of clinical isolates of Neisseria meningitidis. J Clin Microbiol 39, 3066-3071.

Clarke, S. C., Scott, K. J. \& McChlery, S. M. (2004a). Erythromycin resistance in invasive serotype 14 pneumococci is highly related to clonal type. J Med Microbiol 53, 1101-1103.

Clarke, S. C., Scott, K. J. \& McChlery, S. M. (2004b). Serotypes and sequence types of pneumococci causing invasive disease in Scotland prior to the introduction of pneumococcal conjugate polysaccharide vaccines. J Clin Microbiol 42, 4449-4452.

Coffey, T. J., Dowson, C. G., Daniels, M., Zhou, J., Martin, C., Spratt, B. G. \& Musser, J. M. (1991). Horizontal transfer of multiple penicillin-binding protein genes, and capsular biosynthetic genes, in natural populations of Streptococcus pneumoniae. Mol Microbiol 5, 2255-2260.

Coffey, T. J., Enright, M. C., Daniels, M., Morona, J. K., Morona, R., Hryniewicz, W., Paton, J. C. \& Spratt, B. G. (1998). Recombinational exchanges at the capsular polysaccharide biosynthetic locus lead to frequent serotype changes among natural isolates of Streptococcus pneumoniae. Mol Microbiol 27, 73-83.

Coffey, T. J., Daniels, M., Enright, M. C. \& Spratt, B. G. (1999). Serotype 14 variants of the Spanish penicillin-resistant serotype 9V clone of Streptococcus pneumoniae arose by large recombinational replacements of the cpsA-pbpla region. Microbiology 145, 2023-2031.

Cordeiro, S. M., Bajaksouzian, S., Windau, A., Palavecino, E. L. \& Jacobs, M. R. (2005). Clonality of serotypes of amoxicillin resistant isolates of Streptococcus pneumoniae from 1997 to 2003 in Cleveland. In Abstracts of the 105th General Meeting of the American Society for Microbiology 2005, abstract C-121. Washington, DC: American Society for General Microbiology.

Department of Health (2002a). Extending meningitis $C$ vaccine to 20-24 year olds; pneumococcal vaccine for at-risk 2 year olds, PL/ CMO/2002/1. London: Department of Health.

Department of Health (2002b). Update on immunisation issues, PL/CMO/2002/04. London: Department of Health.

Diggle, M. A. \& Clarke, S. C. (2002). Rapid assignment of nucleotide sequence data to allele types for multi-locus sequence analysis (MLSA) of bacteria using an adapted database and modified alignment program. J Mol Microbiol Biotechnol 4, 515-517.

Enright, M. C. \& Spratt, B. G. (1998). A multilocus sequence typing scheme for Streptococcus pneumoniae: identification of clones associated with serious invasive disease. Microbiology 144, 3049-3060.

Eskola, J., Kilpi, T., Palmu, A. \& 9 other authors (2001). Efficacy of a pneumococcal conjugate vaccine against acute otitis media. $N$ Engl J Med 344, 403-409.

Feil, E. J., Li, B. C., Aanensen, D. M., Hanage, W. P. \& Spratt, B. G. (2004). eBURST: inferring patterns of evolutionary descent among clusters of related bacterial genotypes from multilocus sequence typing data. J Bacteriol 186, 1518-1530.

Gertz, R. E., Jr, McEllistrem, M. C., Boxrud, D. J. \& 8 other authors (2003). Clonal distribution of invasive pneumococcal isolates from children and selected adults in the United States prior to 7-valent conjugate vaccine introduction. J Clin Microbiol 41, 4194-4216.

Hanage, W. P., Kaijalainen, T. H., Syrjänen, R. K., Auranen, K., Leinonen, M., Mäkelä, P. H. \& Spratt, B. G. (2005). Invasiveness of serotypes and clones of Streptococcus pneumoniae among children in Finland. Infect Immun 73, 431-435. 
Henrichsen, J. (1995). Six newly recognized types of Streptococcus pneumoniae. J Clin Microbiol 33, 2759-2762.

Jefferies, J., Clarke, S. C., Diggle, M. A., Smith, A., Dowson, C. \& Mitchell, T. (2003). Automated pneumococcal MLST using liquidhandling robotics and a capillary DNA sequencer. Mol Biotechnol 24, 303-308.

Jefferies, J. M. C., Smith, A., Clarke, S. C., Dowson, C. \& Mitchell, T. J. (2004). Genetic analysis of diverse disease-causing pneumococci indicates high levels of diversity within serotypes and capsule switching. J Clin Microbiol 42, 5681-5688.

Kyaw, M. H., Christie, P., Clarke, S. C., Mooney, J. D., Ahmed, S., Jones, I. G. \& Campbell, H. (2003). Invasive pneumococcal disease in Scotland, 1999-2001: use of record linkage to explore associations between patients and disease in relation to future vaccination policy. Clin Infect Dis 37, 1283-1291.

McChlery, S. M., Scott, K. J. \& Clarke, S. C. (2005). Clonal analysis of invasive pneumococcal isolates in Scotland and coverage of serotypes by the licensed conjugate polysaccharide pneumococcal vaccine: possible implications for UK vaccine policy. Eur J Clin Microbiol Infect Dis 24, 262-267.

McEllistrem, M. C., Adams, J. M., Shutt, K., Sanza, L. T., Facklam, R. R., Whitney, C. G., Jorgensen, J. H. \& Harrison, L. H. (2005). Erythromycin-nonsusceptible Streptococcus pneumoniae in children, 1999-2001. Emerg Infect Dis 11, 969-972.

McGee, L., McDougal, L., Zhou, J. \& 8 other authors (2001). Nomenclature of major antimicrobial-resistant clones of Streptococcus pneumoniae defined by the pneumococcal molecular epidemiology network. J Clin Microbiol 39, 2565-2571.

Meats, E., Brueggemann, A. B., Enright, M. C., Sleeman, K., Griffiths, D. T., Crook, D. W. \& Spratt, B. G. (2003). Stability of serotypes during nasopharyngeal carriage of Streptococcus pneumoniae. J Clin Microbiol 41, 386-392.
Miller, E., Waight, P., Efstratiou, A., Brisson, M., Johnson, A. \& George, R. (2000). Epidemiology of invasive and other pneumococcal disease in children in England and Wales 1996-1998. Acta Paediatr Suppl 89, 11-16.

Nesin, M., Ramirez, M. \& Tomasz, A. (1998). Capsular transformation of a multidrug-resistant Streptococcus pneumoniae in vivo. J Infect Dis 177, 707-713.

Pai, R., Gertz, R. E., Whitney, C. G. \& Beall, B. (2005). Clonal association between Streptococcus pneumoniae serotype 23A, circulating within the United States, and an internationally dispersed clone of serotype 23F. J Clin Microbiol 43, 5440-5444.

Smart, L. E. (1986). Serotyping of Streptococcus pneumoniae strains by coagglutination. J Clin Pathol 39, 328-331.

Spratt, B. G. \& Greenwood, B. M. (2000). Prevention of pneumococcal disease by vaccination: does serotype replacement matter? Lancet 356, 1210-1211.

Stefanelli, P., Fazio, C., Neri, A., Sofia, T. \& Mastrantonio, P. (2003). First report of capsule replacement among electrophoretic type 37 Neisseria meningitidis strains in Italy. J Clin Microbiol 41, 5783-5786.

Stephens, D. S., Zughaier, S. M., Whitney, C. G. \& 8 other authors (2005). Incidence of macrolide resistance in Streptococcus pneumoniae after introduction of the pneumococcal conjugate vaccine: population-based assessment. Lancet 365, 855-863.

Swartley, J. S., Marfin, A. A., Edupuganti, S., Liu, L.-J., Cieslak, P., Perkins, B., Wenger, J. D. \& Stephens, D. S. (1997). Capsule switching of Neisseria meningitidis. Proc Natl Acad Sci U S A 94, 271-276.

Whitney, C. G., Farley, M. M., Hadler, J. \& 10 other authors (2003). Decline in invasive pneumococcal disease after the introduction of protein-polysaccharide conjugate vaccine. $N$ Engl J Med 348, $1737-1746$. 